\title{
High Emotional Arousal Enables Subliminal Detection of Concealed Information
}

\author{
Akemi Osugi ${ }^{1,2}$, Hideki Ohira² \\ ${ }^{1}$ Forensic Science Laboratory, Hyogo Prefectural Police Headquarters, Kobe, Japan \\ ${ }^{2}$ Department of Psychology, Graduate School of Environmental Studies, Nagoya University, Nagoya, Japan \\ Email: ak.vermillion74@gmail.com
}

How to cite this paper: Osugi, A., \& Ohira, H. (2017). High Emotional Arousal Enables Subliminal Detection of Concealed Information. Psychology, 8, 1482-1500.

https://doi.org/10.4236/psych.2017.810098

Received: May 25, 2017

Accepted: August 1, 2017

Published: August 4, 2017

Copyright $\odot 2017$ by authors and Scientific Research Publishing Inc. This work is licensed under the Creative Commons Attribution International License (CC BY 4.0).

http://creativecommons.org/licenses/by/4.0/

\begin{abstract}
The Concealed Information Test (CIT) is an information-detecting technique for criminal investigations. Although it has been shown that emotional arousal plays a specific role in the CIT, the mechanisms by which emotional arousal affects the CIT are unclear. The main purpose of this study was to elucidate the processing pathway for stimuli encoded with emotional arousal in a mock crime before the CIT. In this study, participants viewed emotionally arousing pictures before the mock crime. Participants were assigned randomly to either a high or low emotional arousal group, viewing pictures expected to arouse emotion at a high or low level, respectively. Subsequently, all participants enacted the same mock crime, in which they were instructed to stab a pillow with a sharp-edged tool (e.g., kitchen knife or ice pick) as if to harass a woman lying on a bed. After the antecedent emotional experience, a P300-based CIT was conducted using subliminal and supraliminal presentation methods. The results revealed a significantly greater CIT effect on the P300 event-related potential (ERP) component in the High Arousal group compared with the Low Arousal group, under both subliminal and supraliminal conditions. The detection of concealed information was successful only in the High Arousal group under subliminal conditions, whereas detection was successful regardless of the emotional arousal group under supraliminal conditions. These results provide strong evidence that emotional arousal can increase P300 amplitude during responses to concealed information in the CIT. This suggests that concealed information may be automatically processed via the bottom-up route in the CIT, but only when it is encoded with high emotional arousal.
\end{abstract}

\section{Keywords}

Emotional Arousal, Concealed Information Test, Subliminal Presentation, Event Related Potential, P300 


\section{Introduction}

The Concealed Information Test (CIT) has been extensively studied as a technique for detecting information, particularly crime-related memory. Although numerous studies have demonstrated that emotional arousal strongly relates to the formation of memory (Bradley, Greenwald, Petry, \& Lang, 1992; Christianson, 1992; Cahill \& McGaugh, 1998; McGaugh, 2004), little empirical attention has focused on the role of emotional arousal during memory encoding in the CIT.

Osugi \& Ohira (under review) investigated the effects of emotional arousal in the CIT by assessing the amplitudes of the P300 event-related potential (ERP) component. The results revealed that significantly larger P300 amplitudes were elicited in response to concealed items (probe), compared with irrelevant items, in participants who viewed highly emotionally arousing pictures before a mock crime (High Arousal group), compared with participants who viewed pictures associated with relatively low levels of arousal (Low Arousal group). These findings suggest that emotional arousal plays a key role in enhancing the CIT effect, as demonstrated by the difference scores between the probe and irrelevant stimuli, and detection ability in the CIT. However, the mechanisms by which emotional arousal enhances P300 amplitudes in the CIT remain unclear.

There are at least two possibilities for the mechanisms underlying this phenomenon. First, emotional arousal during memory encoding may enhance P300 responses to the probe via the bottom-up processing route in the CIT. Alternatively, emotional arousal may need to be processed via the top-down route to enhance P300 in the CIT. Top-down processes are knowledge-driven mechanisms that enhance the neuronal processing of relevant sensory input, facilitating discrimination between signal and noise; in contrast, bottom-up processes are stimulus-driven functions, which are largely determined by the sensory salience of the stimulus (for a review, see Sarter, Givens, \& Bruno, 2001).

Recent reports have explained the CIT using orienting response (OR) theory (Verschuere, Ben-Shakhar, \& Meijer, 2011) and inhibition theory (Verschuere, Crombez, Koster, Van Bockstaele, \& De Clercq, 2007).The different theories accounting for the CIT effect are based on different physiological measures (Klein Selle, Verschuere, Kindt, Meijer, \& Ben-Shakhar, 2016, 2017). However, it is unclear whether the P300 component in the CIT reflects the orienting response or inhibition (Donchin, Heffley, Hillyard, Loveless, Maltzman, Öhman, Rosler, Ruchkin, \& Siddle, 1984). Several previous studies suggested that the CIT effect on the P300 was caused by OR, without inhibition (Matsuda, Nittono, \& Ogawa, 2013; Rosenfeld, Ozsan, \& Ward, 2017) in line with the electrodermal measuresused by Klein Selle et al. $(2016,2017)$. However, another study reported no CIT effect without inhibition (Kubo \& Nittono, 2009) in accord with the heart rate and respiration measures of Klein Selle et al. (2016, 2017). Because inhibition is defined as an executive function that enables an individual to deliberately and intentionally inhibit a dominant, automatic, prepotent response (Miyake, Frie- 
dman, Emerson, Witzki, Howerter, \& Wager, 2000), it would not be possible to obtain the CIT effect without conscious, top-down processes if inhibition plays a crucial role in the $\mathrm{P} 300$. On the other hand, because the OR is evoked by stimulus novelty and stimulus significance (Verschuere \& Ben-Shakhar, 2011), it may be possible to obtain the CIT effect without conscious, that is, only bottom-up processes if the OR is responsible for the enhancement of the P300.

Previous research investigating stimulus processing using subliminal presentation methods may be useful for resolving these questions. Lui \& Rosenfeld (2009) first applied subliminal presentation in the CIT using the P300, demonstrating a subliminal priming effect on CIT performance. However, because the priming protocol differed from the typical CIT paradigm, it remains unclear whether probe stimuli are automatically recognized and elicit responses through bottom-up processing in the CIT. Maoz, Breska, \& Ben-Shakhar (2012) examined subliminal presentation using the skin conductance response (SCR), in which the probe itself was presented subliminally. The results suggested that probe stimuli might be processed automatically in the CIT. However, the probe in their study was the participant's own name, a type of information that is thought to be processed differently from other information (Yang, Wang, Gu, Gao, \& Zhao, 2013). In the CIT, and in the field, crime-related stimuli are typically used, rather than self-name stimuli. Thus, it remains unclear whether crime-related stimuli are also automatically recognized in the CIT, like selfname stimuli. Previous studies have demonstrated that highly emotionally arousing stimuli elicit more pronounced P300 waves than less emotionally arousing stimuli, even when these stimuli are presented without conscious recognition (Feng, Wang, Liu, Zhu, Dai, Mai, \& Luo, 2012; Rozenkrants, Olofsson, \& Polich, 2008). In line with these studies, a crime-related probe might also be processed via the bottom-up route if it is perceived unconsciously. A clear demonstration of this possibility may elucidate the mechanisms driving the CIT effect on the P300. Such a demonstration may also contribute to the application of this method in the field, potentially enabling the selection of more effective probes (for detailed information about the selection of probes, see (Osugi, 2011)), and the development of methods for countering the countermeasures without special protocols (see (Ben-Shakhar, 2011) and (Rosenfeld, 2011) for detailed information about countermeasures and special protocols).

Here we investigated the effects of emotional arousal on P300 wave amplitudes during the CIT under subliminal conditions. The definition of subliminal, and the objective test used, are described by Maoz et al. (2012). Here subliminal refers to a method in which the participants were aware that a stimulus was presented but were unable to discriminate which stimulus was presented. We determined the absence of correct discrimination using a two-alternative forced choice task (2AFC), confirming that the stimuli were subliminal via comparison with chance-level performance. To compare the results with a previous supraliminal CIT study by Osugi \& Ohira (under review), a suprali- 
minal condition was also used in this study. We followed the same procedure for the manipulation of emotional arousal as Osugi \& Ohira (under review). We hypothesized that the results obtained from both the supraliminal and subliminal conditions would replicate and extend those reported by Osugi \& Ohira (under review).

\section{Method}

\subsection{Participants}

Twenty-six undergraduates (16 male, 10 female) volunteered to participate in this experiment. Their mean age was 20.23 years (range 18 - 29). All participants were right-handed and had normal or corrected vision and did not have any self-reported history of neurological disease. Participants were recruited from psychology classes at a local university, and were given monetary rewards for participating. Participants were randomly assigned to either a high emotional arousal group (High Arousal group) or a low emotional arousal group (Low Arousal group). This study was approved by the ethical committee of Nagoya University, and written informed consent was obtained from each participant prior to the experiment.

\subsection{Manipulation of Emotional Arousal}

The International Affective Picture System (IAPS; Lang, Bradley, \& Cuthbert, 1999) is a standardized collection of color pictures intended to arouse emotion. This has been rated by large groups of North-American participants for valence, dominance, and arousal. The IAPS was used to manipulate the participants' emotional arousal in the same way as described in Osugi \& Ohira (under review). Ten highly emotionally arousing pictures (High Arousal pictures) and 10 low emotionally arousing pictures (Low Arousal pictures) ${ }^{1}$ were selected such that the mean arousal scores were significantly greater in the high emotionally arousing pictures condition than in the low emotionally arousing pictures condition $(t(9)=12.573, p<0.001, d=4.85, B F=21,751,056)^{2}$. The mean valence scores were not significantly different between high and low emotionally arousing pictures $(t(9)=-0.935, p=0.366, d=-0.32, B F=1.85)$, whereas the mean scores differed in the dominance dimension $(t(9)=-2.444, p=0.037, d=-1.21$, $B F=2.70)$. Arousal and valence are the most important dimensions for this manipulation, because they are considered to capture the global and basic elements of emotion. Thus, it is likely that the difference in the dominance dimension has little influence on this manipulation. All pictures were unrelated to a mock crime task in the present study, and were used only for manipulation of emo-

\footnotetext{
${ }^{1}$ The IAPS slide numbers were as follows: high emotionally arousing pictures 3005.1, 3400, 5972, $6260,6550,6831,9300,9600,9635.1$, and 9921; low emotionally arousing pictures $2205,2276,2455$, $3300,9000,9220,9280,9331,9340$, and 9342 .

${ }^{2}$ The mean arousal, valence, and dominance scores were as follows: high emotionally arousing pictures, $6.45,2.42$, and 3.29 , respectively; low emotionally arousing pictures, $4.39,2.58$, and 4.01 , respectively.
} 
tional arousal. Each picture was projected for $10 \mathrm{~s}$ on a cathode ray tube (CRT) display situated $1 \mathrm{~m}$ in front of the participants' eyes. High Arousal pictures were presented to the High Arousal group, and Low Arousal pictures were presented to the Low Arousal group. The participants were told that 10 pictures would be presented and that they should attend to each picture during the entire time it appeared on the screen.

\subsection{Mock Crime}

The mock crime procedure was also the same as that in Osugi \& Ohira (under review). All participants were asked to choose one of five envelopes, each containing two keywords written on a sheet of paper. Keyword 1 was the name of a sharp-edged tool to be used in the mock crime (i.e., kitchen knife, box-cutter, ice pick, sickle, or saw). Keyword 2 was "pillow" in every envelope and was to be stabbed by the participants in both emotional arousal groups with the object described by Keyword 1. Unknown to the participants, Keyword 1 items were counterbalanced, with each keyword arranged in order of participation, for each arousal group.

After choosing one of five envelopes, each participant was asked to thoroughly memorize the two keywords for 1 minute. All participants then engaged in a recall test, in which they wrote the two keywords five times to confirm and reinforce their memory. This procedure was not realistic, but was designed to ensure that participants remembered the probe, because the purpose of the current study was to investigate the effects of emotional arousal, and lack of recognition of the probe caused by forgetting would contaminate the effects.

All participants then enacted the mock crime. Participants were instructed to move to a separate room by themselves and look for the item indicated by Keyword 1. After finding it, they were instructed to stab Keyword 2 (pillow) with the Keyword 1 item a couple of times, as forcefully as possible, as if to harass a female mannequin lying on a bed. The participants were also instructed to stay in the room for more than 10 minutes and to smuggle the Keyword 1 item out of the room, keeping the item in their bag carefully covered with a towel.

\subsection{CIT}

The P300-based CIT was then administered, and participants were told that the experiment was designed to check whether they had remembered Keyword 1, which was the sharp-edged tool used in the mock crime. They were also asked to pretend to be innocent and to make an effort to avoid positive detection by electroencephalogram (EEG). Motivational instructions were given; participants were told that if they were not detected they would receive a monetary reward. All participants were instructed that there were two conditions in this experiment; one in which they could see the stimuli easily (supraliminal condition), and one in which they could not (subliminal condition). The details of these conditions are described in the section below. Before commencing the CIT, the participants took 
part in a practice session for both conditions using six pictures of items of stationery. The order of these conditions was counterbalanced. In the practice session, the participants chose one of five cards, and memorized the picture on the card. They were then required to press the left button in response to a picture of scissors as the target, and the right button to the other pictures; ballpoint pen, ruler, staple, correction tape dispenser, and glue stick. The practice session consisted of 36 trials for each condition. In the CIT, six pictures of the sharp-edged tool were presented on the CRT at a constant interval representing the question: "Did you use this tool in the mock crime?" They were required to press the button quickly and accurately with the right hand as the stimulus was presented, to indicate "No". To ensure that participants attended to the stimuli and performed the stimulus classification prerequisite for the elicitation of the $\mathrm{P} 300$, they were asked to press the left button only to the target stimulus. They were also instructed to press the right button when the other five pictures were presented, which included a probe and irrelevant stimuli. The buttons were counterbalanced between participants. The probe was a picture of the tool the participant used in the mock crime, and differed between participants. The other four pictures were irrelevant stimuli, which were not related to the mock crime.

\subsubsection{Stimulus Presentation}

Six $12.9 \times 10.7 \mathrm{~cm}$ pictures of sharp-edged tools (i.e., scissors, kitchen knife, box-cutter, ice pick, sickle, and saw), were used as stimuli for the CIT. A picture of scissors was always used as the target stimulus, and the others were probe or irrelevant stimuli. The pictures were projected on a CRT display situated $1 \mathrm{~m}$ in front of the participants. Each trial began with a red fixation cross, presented in the middle of the screen for $1000 \mathrm{~ms}$, and a gray fixation cross followed. After presentation of the gray fixation cross for $1000 \mathrm{~ms}$, a pre-mask stimulus was presented for $15 \mathrm{~ms}$. The following protocol differed in each condition as shown in Figure 1. In the supraliminal condition, all pictures of edged tools were presented for $300 \mathrm{~ms}$ after the pre-mask stimulus. This duration was enough for all participants to recognize the presented stimulus well. The gray fixation cross was presented for $1000 \mathrm{~ms}$ again after each picture of a sharp-edged tool. In the subliminal condition, only the target stimulus was presented for $70 \mathrm{~ms}$, and the others for $30 \mathrm{~ms}$. All tool pictures were masked by pre- and post-masks, which were presented for $230 \mathrm{~ms}$ for the target and $270 \mathrm{~ms}$ for the other stimuli. Participants could just recognize the target, but otherwise could not distinguish which sharpedged tool picture was presented. The gray fixation cross was presented for 1000 ms once again after the tool picture, as in the supraliminal condition. The stimulus onset asynchrony (SOA) between each picture of a sharp-edged tool was $3315 \mathrm{~ms}$ in both conditions. Participants were instructed not to blink when the tool picture and gray fixation cross were presented. Presentation software (Neurobehavioral Systems, Inc.) controlled the stimulus presentation via a personal computer (OPTIPLEX980; Dell Inc.). In both conditions, the target and probe stimuli were each presented 48 times, and the irrelevant stimuli were presented 


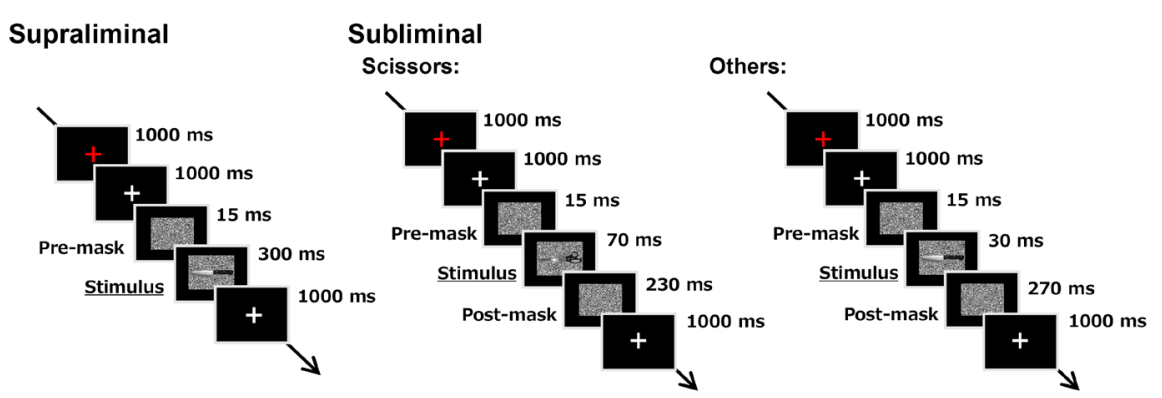

Figure 1. Timing of mask and stimulus presentations in each condition.

192 times, in a random order over four sessions. Between sessions, the participants took a 2-min break. Each condition was conducted alternately for each session, and the order was randomized among participants.

\subsubsection{Indices}

In the CIT, EEG data were recorded. EEG data were recorded from 20 sites (Fp1/2, F3/4, F7/8, Fz, C3/4, T7/8, Cz, P3/4, P7/8, Pz, O1/2, and Oz) according to the extended $10-20$ system. The reference electrode was placed on the nose and the forehead was grounded. Electrooculograms (EOG) were also recorded from electrodes placed supra-orbitally of the left eye. Electrocap (EASYCAP Inc.) and $\mathrm{Ag} / \mathrm{AgCl}$ electrodes were used. Electrode impedance did not exceed $5 \mathrm{k} \Omega$. Signals were amplified by Neurofax (Nihon Kohden, Inc.) with a $30 \mathrm{~Hz}$ high-cut filter and $0.1 \mathrm{~Hz}$ low-cut filter. Amplified EEG and EOG signals were digitized at a rate of 500 points per second and recorded. After recording, EEG data for 1200 $\mathrm{ms}$, from $200 \mathrm{~ms}$ prior to stimulus onset to $1000 \mathrm{~ms}$ after stimulus onset, were analyzed. The average amplitude from 200 - $15 \mathrm{~ms}$ pre-stimulus was used as a baseline. In the averaging procedure, epochs in which the signal amplitudes exceeded $\pm 80 \mu \mathrm{V}$ on any of the electrodes were removed.

\subsection{Two-Alternative Forced Choice Task}

To determine the lack of conscious awareness of each stimulus in the subliminal condition, a two-alternative forced choice task (2AFC) was conducted. Excluding the picture of scissors, five pictures of the tools used in the CIT (i.e., kitchen knife, box-cutter, ice pick, sickle, and saw) and five pictures of stationery used in the practice session (i.e., ballpoint pen, ruler, staple, correction tape dispenser, and glue stick) were used. Each stimulus was presented for $30 \mathrm{~ms}$ between preand post-masks, as in the subliminal condition, with the exception of the scissors, as shown in Figure 1. The stimuli were projected on a CRT display situated $1 \mathrm{~m}$ from the participants. All participants were required to judge whether the presented stimulus was a sharp-edged tool or an item of stationery. They pressed the left button for tools and the right button for stationery using the right hand as quickly as possible after the stimulus was presented. They were instructed that they had to press the left or right button without explicit consideration, even though they could not distinguish which picture was presented, and not to con- 
tinually press only one button. It was assumed that lack of perception of the pictures would result in chance-level performance. The buttons were counterbalanced. Each stimulus was presented 10 times in random order, with $1002 \mathrm{AFC}$ trials in total.

\subsection{Self-Report}

The Japanese version of the University of Wales Institute of Science and Technology (UWIST) Mood Adjective Checklist shortened version (JUMACL; Shirasawa, Ishida, Hakoda, \& Haraguchi, 1999) was used in the same way as described in Osugi \& Ohira (under review). The JUMACL is composed of two subscales of 10 items each; Energetic Arousal (ranging from feeling sleepy to feeling awake) and Tense Arousal (ranging from feeling calm to feeling nervous). These subscales are sensitive to external stressors, and participants scoring high on Energetic Arousal report feeling vigorous, bright, and active, while high Tense Arousal scores imply nervousness, jitters, and tenseness. Matthews, Jones, \& Chamberlain (1990) reported that these two scales have different features and only Tense Arousal is raised by both unpleasant physical and psychological stressors, so only Tense Arousal was used in this study (see Oue, Hakoda, Onuma, \& Morikawa, 2001, for the same analysis). The participants were instructed to rate the applicability of each adjective to their present mood on a four-choice symmetric format, as "definitely", "slightly", "slightly not", and "definitely not". Responses were scored from 4 for "definitely" to 1 for "definitely not".

\subsection{Procedure}

All participants were informed before starting the experiment that they would be required to enact a mock crime and take the CIT using ERPs. After instructions were given, emotional arousal was manipulated for each arousal group. All participants then enacted the mock crime task. After attaching the physiological assessment equipment, the CIT was conducted. Following the CIT, the 2AFC task was performed.

All participants were required to make subjective ratings of their emotional arousal using the JUMACL at five periods; before and after the manipulation of emotional arousal, after the mock crime, and before and after the CIT. At the end of the experiment a short questionnaire was administered to ask participants how they felt emotionally during the mock crime and the CIT, and how they recognized the stimuli presented in both conditions. We also confirmed whether they consciously recognized the stimuli in the subliminal condition and remembered the probe.

\subsection{Analysis}

In the analyses, target stimuli were excluded because the target was different to the probe and irrelevant stimuli. That is, participants pressed different buttons in the CIT and only recognized the target in the subliminal condition because of 
its different duration. In addition, the focus of this study was not on the target but on the differences between the probe and irrelevant stimuli (see Matsuda, Nittono, \& Allen, 2013, for a similar analysis).

We calculated JUMACL scores and difference scores of P300 amplitudes between the probe and the irrelevant stimuli, and conducted repeated measures analysis of variance (ANOVA) for each. ANOVA $F$-statistics are positively biased, and the risk of a Type I error increases when repeated-measures factors have more than two levels, as is the case here with periods. Thus, the $p$-values of all effects resulting from these factors were corrected towards conservative interpretation by reducing the degrees of freedom when Mauchly's test of sphericity was violated. This correction was performed by multiplying the original degrees of freedom by the Greenhouse-Geisser epsilon. Bonferroni correction was used for post hoc comparisons in all cases. Follow-up $t$-tests were also conducted for the P300 amplitudes to confirm each CIT effect. ANOVA effect sizes were calculated using partial eta squared $\left(\eta p^{2}\right)$ and those in $t$-tests were obtained using Cohen's $d$. In addition, to confirm the discrimination performance of the P300 amplitudes, a receiver operating characteristic (ROC) curve was calculated for each group and in each condition. The area under the ROC curve is an index of detection efficiency (see National Research Council, 2003). Because a sample of unknowledgeable (innocent) participants was not included in this study, the expected detection score distribution among unknowledgeable participants was simulated using the method proposed by Meijer, Smulders, Johnston, \& Merckelbach (2007). These calculations were performed using PASW Statistics 18 (IBM). Calculation of the Bayes factors was performed using JASP (JASP team, 2016). Data from two participants were discarded due to low correct answer rates in the CIT (less than $80 \%$ ), leaving a final sample of 24 participants (High Arousal group; $n=12$, Low Arousal group; $n=12$ ).

\section{Results}

\subsection{Manipulation Check}

\subsubsection{Arousal Check}

The mean scores and standard errors for Tense Arousal are shown in Figure 2. The mean arousal scores at period before and after the manipulation of emotional arousal were $17.75(\mathrm{SD}=1.14)$ and $24.08(\mathrm{SD}=0.82)$ in the High Arousal group, and were $18.41(\mathrm{SD}=1.54)$ and $18.08(\mathrm{SD}=1.70)$ in the Low Arousal group. The mean arousal score at period after the mock crime was 31.66 (SD = $1.09)$ in the High Arousal group and $27.66(\mathrm{SD}=1.57)$ in the Low Arousal group. The mean arousal scores at period before and after the CIT were $18.00(\mathrm{SD}=1.59)$ and $17.41(\mathrm{SD}=0.67)$ in the High Arousal group, and $15.58(\mathrm{SD}=1.09)$ and $17.16(\mathrm{SD}=1.29)$ in the Low Arousal group. A Group (High, Low) $\times$ Period (before and after the manipulation of emotional arousal, after the mock crime, and before and after the CIT) ANOVA was conducted. The analysis revealed a significant main effect of Period $\left(F[4,88]=55.032, p<0.001, \eta p^{2}=0.714\right)$ and a 


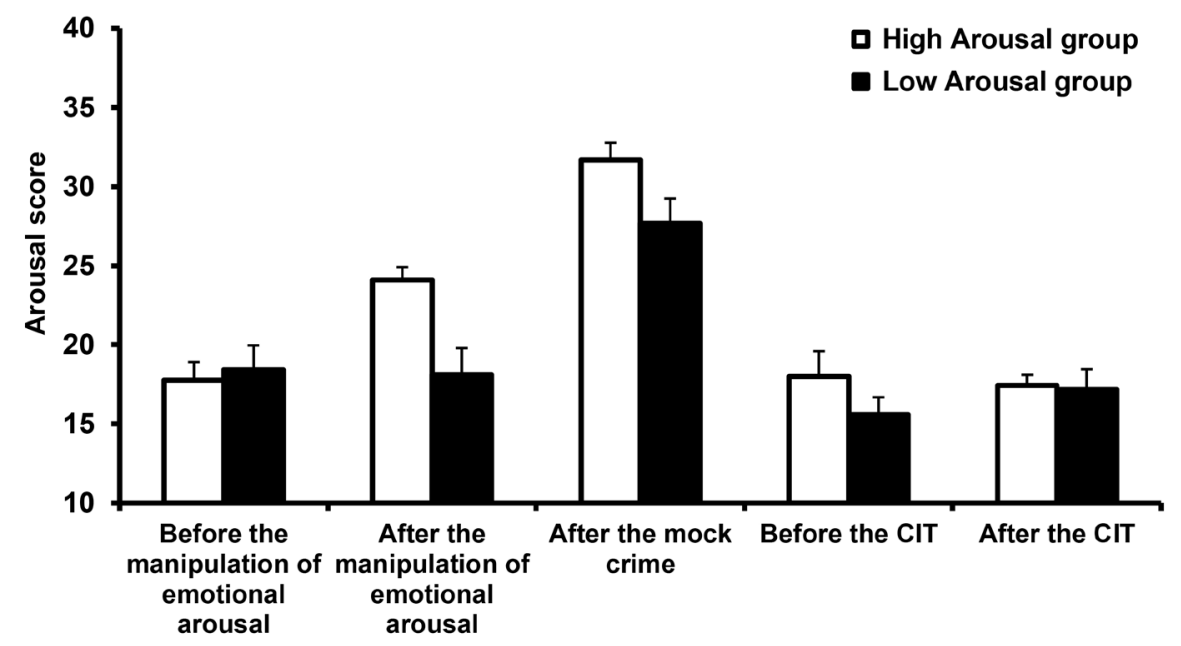

Figure 2. Mean scores of Tense Arousal in each group in each period. Error bars indicate standard errors.

significant interaction of Group $\times$ Period $\left(F[4,88]=3.559, p=0.01, \eta p^{2}=0.139\right)$. According to post hoc comparisons, Tense Arousal scores were significantly higher in the High Arousal group than in the Low Arousal group after the manipulation of emotional arousal $(p=0.004)$, and after the mock crime $(p=0.049)$, whereas there were no differences between both arousal groups before the manipulation of emotional arousal ( $p=0.732)$, before the CIT $(p=0.224)$, and after the CIT ( $p=0.866)$, indicating that the manipulation of emotional arousal in this study was successful.

Here, in the High Arousal group, Tense Arousal scores were significantly heightened after the manipulation of emotional arousal compared with before the manipulation of emotional arousal $(p<0.001)$, and were also significantly higher after the mock crime than after the manipulation of emotional arousal ( $p$ $<0.001)$. On the other hand, in the Low Arousal group, Tense Arousal was significantly heightened after the mock crime compared with before the manipulation of emotional arousal $(p<0.001)$ and after the manipulation of emotional arousal $(p<0.001)$.

\subsubsection{Awareness Check}

The thresholds reported by Maoz et al. (2012) were applied to confirm that each participant was not aware of the subliminally presented stimuli. We set a lower threshold of $45 \%$ and an upper threshold of $65 \%$, and eliminated participants whose scores were outside these thresholds, because such high or low correct rates indicated that participants were subjectively aware of the stimuli. All correct rates were within these thresholds, and we confirmed that each participant was not subjectively aware of the subliminal stimuli. All data from included participants were used in the subsequent analysis. Participants' self-reports also confirmed that they were unable to recognize which stimulus was presented in the subliminal condition, other than the target stimulus. 


\subsection{CIT}

\subsubsection{ERP Data}

In the following analysis, the target stimulus was excluded because it was different from the other stimuli (i.e., the probe and irrelevant stimuli), in that participants pressed different buttons in the CIT, and the focus of this study was on the differences between the probe and irrelevant stimuli, not on the target stimuli (see Matsuda, Nittono, \& Allen, 2013, for a similar analysis).

Grand averaged ERPs within the Stimulus type categories (probe, irrelevant) for each group in each condition are shown in Figure 3. As prominent positive waves were found in both the High and Low Arousal groups, mainly in the supraliminal condition, we investigated this component as the P300 using the peak-amplitude method (see Osugi \& Ohira (under review), for the same analysis). Several previous studies investigated the P300 at Pz using the peak-peak (p-p) method, which computes the difference between the P300 peak and the bottom peak from the beginning of the P300 latency to approximately $1300 \mathrm{~ms}$ after stimulus onset, and reported that the P300-based CIT method was effective (e.g., Soskins, Rosenfeld, \& Niendam, 2001; Rosenfeld \& Labkovsky, 2010; Rosenfeld, 2011). However, in the present study we used the peak-amplitude method, because the clear negative peaks that are usually obtained following P300 peaks in P300-based CIT studies were not observed. This finding might be explained by previous reports that late positive potentials can be modulated in affective picture processing and motivated attentional processing (Schupp, Cuthbert, Bradley, Cacioppo, Ito, \& Lang, 2000; Matsuda \& Nittono, 2015). In addition, the analysis was confined to $\mathrm{Pz}$, a midline parietal scalp site, as previous P300-based CIT studies reported that the largest effect was shown at $\mathrm{Pz}$

\section{Supraliminal}
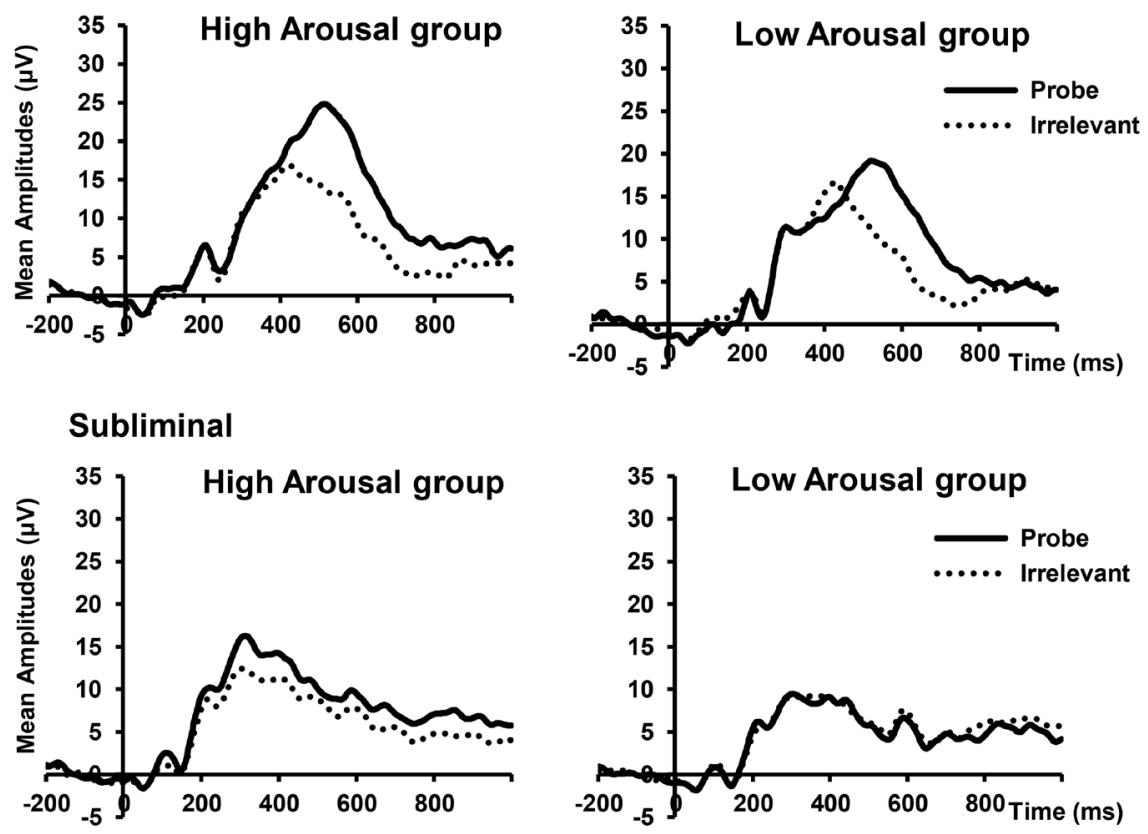

Figure 3. Grand average ERPs at $\mathrm{Pz}$ for each group in both conditions. 
and the researchers limited their analysis to this region (Rosenfeld, Shue, \& Singer, 2007; Rosenfeld \& Labkovsky, 2010). Therefore, we calculated the largest positive peak in the range of $200-700 \mathrm{~ms}$ at $\mathrm{Pz}$.

\subsubsection{P300 Amplitude}

The mean amplitudes at $\mathrm{Pz}$ according to Stimulus type for each group are shown in Figure 4. In the supraliminal condition, the mean amplitude in response to the probe stimuli was $27.45(\mathrm{SD}=1.97)$ in the High Arousal group and 21.11 $(\mathrm{SD}=1.98)$ in the Low Arousal group, and the mean amplitude to the irrelevant stimuli was $18.68(\mathrm{SD}=1.70)$ in the High Arousal group and $17.85(\mathrm{SD}=1.95)$ in the Low Arousal group. In the subliminal condition, the mean amplitude in response to the probe stimuli was $17.64(\mathrm{SD}=1.70)$ in the High Arousal group and 11.95 ( $\mathrm{SD}=1.75)$ in the Low Arousal group, and the mean amplitude to the irrelevant stimuli was $14.28(\mathrm{SD}=1.35)$ in the High Arousal group and 11.86 $(\mathrm{SD}=1.56)$ in the Low Arousal group. Moreover, we calculated the difference scores of P300 amplitude between the probe and the irrelevant stimuli according to the Presentation condition in each group. In the supraliminal condition, the difference score was $8.78(\mathrm{SD}=1.05)$ in the High Arousal group and $3.25(\mathrm{SD}=$ 0.55) in the Low Arousal group, whereas in the subliminal condition the difference score was $3.35(\mathrm{SD}=0.51)$ in the High Arousal group and $0.09(\mathrm{SD}=0.92)$ in the Low Arousal group. A two-way ANOVA was used to compare the difference score with Group (High, Low) $\times$ Presentation condition (supraliminal, subliminal). We found a significant main effect of $\operatorname{Group}(F[1,22]=32.772, p<$ $\left.0.001, \eta p^{2}=0.598, B F=40.574\right)$ and a significant main effect of Presentation condition $\left(F[1,22]=27.04, p<0.001, \eta p^{2}=0.551, B F=1987.592\right)$. These results showed that the CIT effect was significantly greater in the High Arousal group than in the Low Arousal group, and also significantly greater in the supraliminal condition than in the subliminal condition, regardless of group. A Group $\times$ Presentation condition interaction was not detected $(F[1,22]=15.388, p=$ $\left.0.184, \eta p^{2}=0.079, B F=543257.797\right)$.
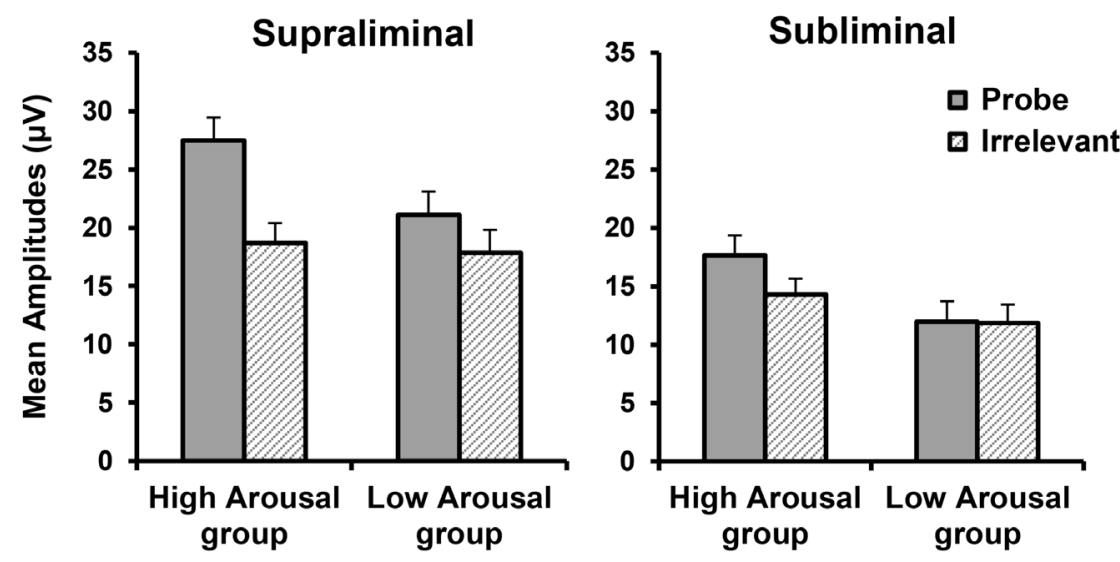

Figure 4. Mean amplitudes according to Stimulus Type in each group. Error bars indicate standard errors. 
As a follow-up analysis, we first confirmed the CIT effect for each condition for each group by within-subject $t$-test of the differentiation between the probe and irrelevant stimuli together with Bayesian hypothesis testing. The $t$-tests revealed that, in the supraliminal condition, P300 amplitudes in response to the probe were significantly larger than those to irrelevant stimuli in both groups (High Arousal group; $t[11]=8.322, p<0.001, d=2.402, B F=4387.52$, Low Arousal group; $t[11]=5.829, p<0.001, d=1.683, B F=256.927)$. In the subliminal condition, P300 amplitude in response to the probe was also significantly greater than those in response to irrelevant stimuli in the High Arousal group $(t$ $[11]=6.506, p<0.001, d=1.878, B F=589.613)$, although the amplitude in response to the probe in the Low Arousal group was not significantly different to responses to the irrelevant stimuli $(t[11]=0.099, p=0.923, d=0.029, B F=$ 0.289 ). In addition, the difference score in the supraliminal condition was significantly higher in the High Arousal group than in the Low Arousal group ( $t$ $[22]=4.632, p<0.001, d=1.891, B F=164.2)$, which is consistent with that reported by Osugi \& Ohira (under review). Although the Bayes factor was not strong, the difference score was significantly higher in the High Arousal group than in the Low Arousal group in the subliminal condition $(t$ [22] $=3.088, p=$ $0.005, d=1.261, B F=8.192$ ).

Comparing the detection efficiency of the present results with previous CIT studies, we calculated a receiver operating characteristic (ROC) curve as described by Meijer, et al. (2007) and the area under the ROC curve (AUC). Table 1 shows the AUC with a corresponding 95\% confidence interval (CI) for each group in each condition.

\section{Discussion}

The present study aimed to investigate whether stimuli encoded with emotional arousal are processed via the top-down or bottom-up route in the CIT. To this end, we tested participants on the CIT under both supraliminal and subliminal conditions. Consistent with our hypothesis, the CIT effect on P300 amplitude was significantly greater in the High Arousal group than in the Low Arousal group, under both conditions. However, the detection of the probe was successful only in the High Arousal group under the subliminal condition, while detection was successful in both groups under the supraliminal condition.

Importantly, the results revealed that the CIT effect was markedly larger in the High Arousal group than in the Low Arousal group in both presentation

Table 1. Area under the Receiver Operating Characteristic (ROC) curve (AUC) with $95 \%$ confidence interval (CI) of P300 amplitudes for each group and in each condition.

\begin{tabular}{ccc}
\hline & Supraliminal condition & Subliminal condition \\
\hline $\begin{array}{c}\text { High Arousal group } \\
\text { AUC }(95 \% \text { CI })\end{array}$ & $0.743(0.541,0.945)$ & $0.646(0.419,0.872)$ \\
Low Arousal group & & \\
AUC $(95 \%$ CI $)$ & $0.639(0.413,0.865)$ & $0.563(0.328,0.797)$ \\
\hline
\end{tabular}


conditions. These results provide strong evidence that emotional arousal can influence the detection efficiency of the P300 in the CIT. These results are in strong agreement with Osugi \& Ohira (under review), in which it was first demonstrated that emotional arousal significantly increases the differentiation between the P300 responses to the probe and irrelevant stimuli in the supraliminal CIT. In this study, we obtained the same results under the subliminal condition. Leiphart, Rosenfeld, \& Gabrieli (1993) reported that P300 amplitude was greater for subliminally presented old than new words, and was no different between high emotional and low emotional words in the subliminal condition. However, because they did not select the emotional words based on arousal ratings, it is unclear whether the current results are consistent with this previous finding. Focused on emotional arousal, some studies have demonstrated that highly emotionally arousing stimuli elicit more pronounced P300 waves than low emotionally arousing stimuli, even when these stimuli are presented without awareness (Feng, Wang, Liu, Zhu, Dai, Mai, \& Luo, 2012; Rozenkrants, Olofsson, \& Polich, 2008). These previous findings are in accord with the current study, confirming that emotional arousal increases P300 responses to probes in the CIT, regardless of conscious awareness.

Second, the probe encoded with high emotional arousal could be detected under the subliminal condition, while probe encoded with low emotional arousal could not. This is the first report of subliminal effects in the typical CIT paradigm as assessed using P300 parameters, except for one study using the priming method (Lui \& Rosenfeld, 2009). This suggests that the probe may be automatically processed via the bottom-up route in the CIT, but only when the probe was encoded with high emotional arousal. Under the subliminal condition the participants could not be discriminate which stimulus was presented, confirming that the subliminal stimuli were processed without conscious awareness. Thus, the CIT effect on the P300 under these conditions was presumably derived from stimulus-driven processing, suggesting that emotional arousal may have increased the significance of the stimulus at encoding, potentially causing a greater CIT effect in the context of the OR. Several previous studies demonstrated that aversive masked stimuli can elicited greater skin conductance responses than neutral stimuli (Van den Hout, De Jong, \& Kindt, 2000; Silvert, Delplanque, Bouwalerha, Verpoort, \& Sequeira, 2004), suggesting that the OR could be elicited only via bottom-up processing without conscious awareness.

Another possible explanation is related to the dual route model proposed by LeDoux (1996). According to this model, emotional stimuli are processed using two parallel pathways to the amygdala from the sensory thalamus: the Low road, a "quick and dirty" subcortical pathway for transferring rapid activity directly to the amygdala; and the High road, providing slower but highly processed cortical information. The probe in the CIT may, with time, be processed via the High road when the probe is associated with relatively low emotional arousal. However, only a probe encoded with high emotional arousal may be quickly processed 
via the Low road, independent of the top-down cortical loop, as threatening or fear-inducing stimuli are processed automatically. Because this is specific to emotionally-relevant stimuli, this interpretation is speculative and may not generalize to other types of stimulus in the CIT. However, numerous fMRI studies have shown that subliminal presentation of arousing stimuli automatically induces robust activation in the amygdala (see the meta-analysis by Brooks, Savov, Allzen, Benedict, Fredrilsson, \& Schioth, 2012). The existence of a dual route model is plausible, in which the CIT probe can be detected automatically via the Low road when it is strongly encoded with emotional arousal. Applying this model may lead not only to an additional explanation of the theoretical mechanisms of the CIT, but also has several potential advantages for practical use of the CIT. For example, adding a new component of emotional arousal to the CIT model may enable selection of the most appropriate probe for quick and powerful detection. Furthermore, considering subconscious detection may enable measures for countering the problem of countermeasures, which are examinees' attempts to defeat or distort a test and are one of the limitations of the conventional CIT paradigm, in addition to the modified protocol introduced by Lui \& Rosenfeld (2009), as reported by Rosenfeld (2011).

The current study involved several limitations that should be considered, particularly regarding the number of the participants tested. Although we obtained significant results in terms of the effect of emotional arousal in both conditions, it should be noted that the sample size was relatively low. To examine this effect more rigorously, further research with a larger sample should be conducted in future. Another limitation is related to the absence of a probe-vs.-irrelevant stimulus difference in the subliminal condition in the Low Arousal group. Because the P300 amplitudes in the subliminal condition were generally small for both the probe and irrelevant stimuli, it remains possible that probe stimuli encoded with relatively low emotional arousal were processed via the bottom-up route in the CIT, but not detected in P300 responses. Further experiments using other indices would help to clarify this question. In addition, our manipulation of emotional arousal was checked only by subjective self-report. Future studies measuring physiological responses during encoding would be helpful for confirming the manipulation of emotional arousal more precisely.

It should be noted that this study did not aim to investigate straightforward implementations of the subliminal CIT, although the results provide partial support for the application of the subliminal method. While few previous studies have examined the subliminal method within the CIT model, the present study is the first to examine the effect of emotional arousal in the subliminal CIT by assessing P300 amplitudes. The results revealed that emotional arousal enables powerful detection under supraliminal conditions, and can even aid subliminal detection. These findings provide strong evidence that emotional arousal plays an important role in the CIT, providing new understanding of the CIT and its application. 


\section{Conclusion}

The main purpose of this study was to elucidate the processing pathway for stimuli encoded with emotional arousal in the CIT. We manipulated emotional arousal before a mock crime and investigated its effect on the P300-based CIT using subliminal and supraliminal presentation methods. The results revealed a significantly greater CIT effect on the P300 in the High Arousal group than in Low Arousal group, in both supraliminal and subliminal conditions. These results provide strong evidence that emotional arousal can increase the P300 amplitude of responses to the probe in the CIT. We may reconsider the conventional model of the CIT applying the dual route model. These results suggest that probe stimuli maybe automatically processed via the Low road in the CIT when they are strongly encoded with emotional arousal. These findings extend current understanding of the processing path ways of probe stimuli in the CIT.

\section{Acknowledgements}

We wish to thank the editor and the anonymous reviewers for comments on an earlier version of this paper.

\section{Funding Sources}

This work was supported by JSPS KAKENHI Grant Number JP26906006.

\section{References}

Ben-Shakhar, G. (2011). Countermeasures. In B. Verschuere, G. Ben-Shakhar, \& E. Meijer (Eds.), Memory Detection: Theory and Application of the Concealed Information Test (pp. 200-214). Cambridge: Cambridge University Press. https://doi.org/10.1017/CBO9780511975196.012

Bradley, M. M., Greenwald, M. K., Petry, M. C., \& Lang, P. J. (1992). Remembering Pictures: Pleasure and Arousal in Memory. Journal of Experimental Psychology: Learning, Memory, and Cognition, 18, 379-390. https://doi.org/10.1037/0278-7393.18.2.379

Brooks, S. J., Savov, V., Allzen, E., Benedict, C., Fredriksson, R., \& Schiöth, H. B. (2012). Exposure to Subliminal Arousing Stimuli Induces Robust Activation in the Amygdala, Hippocampus, Anterior Cingulate, Insular Cortex and Primary Visual Cortex: A Systematic Meta-Analysis of FMRI Studies. NeuroImage, 59, 2962-2973. https://doi.org/10.1016/j.neuroimage.2011.09.077

Cahill, L., \& McGaugh, J. L. (1998). Mechanisms of Emotional Arousal and Lasting Declarative Memory. Trends in Neurosciences, 21, 294-299. https://doi.org/10.1016/S0166-2236(97)01214-9

Christianson, S. A. (1992). Emotional Stress and Eyewitness Memory: A Critical Review. Psychological Bulletin, 112, 284-309. https://doi.org/10.1037/0033-2909.112.2.284

Donchin, E., Heffley, E., Hillyard, S. A., Loveless, N., Maltzman, I., Öhman, A., Rosler, F., Ruchkin, D., \& Siddle, D. (1984). Cognition and Event-Related Potentials II. The Orienting Reflex and P300. Annals of the New York Academy of Sciences, 425, 39-57. https://doi.org/10.1111/j.1749-6632.1984.tb23522.x

Elaad, E., \& Ben-Shakhar, G. (1989). Effect of Motivation and Verbal Response Type on Psychophysiological Detection of Information. Psychophysiology, 26, 442-451. 
https://doi.org/10.1111/j.1469-8986.1989.tb01950.x

Feng, C., Wang, L., Liu, C., Zhu, X., Dai, R., Mai, X., \& Luo, Y. J. (2012). The Time Course of the Influence of Valence and Arousal on the Implicit Processing of Affective Pictures. PloS One, 7, e29668. https://doi.org/10.1371/journal.pone.0029668

Klein Selle, N., Verschuere, B., Kindt, M., Meijer, E., \& Ben-Shakhar, G. (2016). Orienting versus Inhibition in the Concealed Information Test: Different Cognitive Processes Drive Different Physiological Measures. Psychophysiology, 53, 579-590.

https://doi.org/10.1111/psyp.12583

Klein Selle, N., Verschuere, B., Kindt, M., Meijer, E., \& Ben-Shakhar, G. (2017). Unraveling the Roles of Orienting and Inhibition in the Concealed Information Test. Psychophysiology, 54, 628-639. https://doi.org/10.1111/psyp.12825

Kubo, K., \& Nittono, H. (2009). The Role of Intention to Conceal in the P300-Based Concealed Information Test. Applied Psychophysiology and Biofeedback, 34, 227-235. https://doi.org/10.1007/s10484-009-9089-y

Lang, P. J., Bradley, M. M., \& Cuthbert, B. N. (1999). International Affective Picture System (IAPS): Instruction Manual and Affective Ratings. Gainesville, FL: The Center for Research in Psychophysiology, University of Florida.

LeDoux, J. E. (1996). The Emotional Brain. New York: Simon \& Schuster.

Leiphart, J., Rosenfeld, J. P., \& Gabrieli, J. D. (1993). Event-Related Potential Correlates of Implicit Priming and Explicit Memory Tasks. International Journal of Psychophysiology, 15, 197-206. https://doi.org/10.1016/0167-8760(93)90003-8

Lui, M., \& Rosenfeld, J. P. (2009). The Application of Subliminal Priming in Lie Detection: Scenario for Identification of Members of a Terrorist Ring. Psychophysiology, 46, 889-903. https://doi.org/10.1111/j.1469-8986.2009.00810.x

Maoz, K., Breska, A., \& Ben-Shakhar, G. (2012). Orienting Response Elicitation by Personally Significant Information under Subliminal Stimulus Presentation: Demonstration Using the Concealed Information Test. Psychophysiology, 49, 1610-1617. https://doi.org/10.1111/j.1469-8986.2012.01470.x

Matsuda, I., \& Nittono, H. (2015). Motivational Significance and Cognitive Effort Elicit Different Late Positive Potentials. Clinical Neurophysiology, 126, 304-313. https://doi.org/10.1016/j.clinph.2014.05.030

Matsuda, I., Nittono, H., \& Allen, J. J. (2013). Detection of Concealed Information by P3 and Frontal EEG Asymmetry. Neuroscience Letters, 537, 55-59. https://doi.org/10.1016/j.neulet.2013.01.029

Matsuda, I., Nittono, H., \& Ogawa, T. (2013). Identifying Concealment-Related Responses in the Concealed Information Test. Psychophysiology, 50, 617-626. https://doi.org/10.1111/psyp.12046

Matthews, G., Jones, D. M., \& Chamberlain, A. G. (1990). Refining the Measurement of Mood: The UWIST Mood Adjective Checklist. British Journal of Psychology, 81, 17-42. https://doi.org/10.1111/j.2044-8295.1990.tb02343.x

McGaugh, J. L. (2004). The Amygdala Modulates the Consolidation of Memories of Emotionally Arousing Experiences. Annual Reviews of Neuroscience, 27, 1-28. https://doi.org/10.1146/annurev.neuro.27.070203.144157

Meijer, E. H., Smulders, F. T., Johnston, J. E., \& Merckelbach, H. L. (2007). Combining Skin Conductance and Forced Choice in the Detection of Concealed Information. Psychophysiology, 44, 814-822. https://doi.org/10.1111/j.1469-8986.2007.00543.x

Miyake, A., Friedman, N. P., Emerson, M. J., Witzki, A. H., Howerter, A., \& Wager, T. D. (2000). The Unity and Diversity of Executive Functions and Their Contributions to 
Complex "Frontal Lobe" Tasks: A Latent Variable Analysis. Cognitive psychology, 41, 49-100. https://doi.org/10.1006/cogp.1999.0734

National Research Council (2003) The Polygraph and Lie Detection. Washington DC: The National Academic Press, Committee to Review the Scientific Evidence on the Polygraph, Division of Behavioral and Social Science and Education.

Osugi, A. (2011). Daily Application of the Concealed Information Test: Japan. In B. Verschuere, G. Ben-Shakhar, \& E. Meijer (Eds.), Memory Detection: Theory and Application of the Concealed Information Test (pp. 253-275). Cambridge: Cambridge University Press. https://doi.org/10.1017/CBO9780511975196.015

Osugi, A., \& Ohira, H. (under Review). Emotional Arousal at Memory Encoding Enhanced P300 in the Concealed Information Test. Frontiers in Psychology.

Oue, W., Hakoda, Y., Onuma, N., \& Morikawa, S. (2001). The Effect of Negative Emotion on Eyewitness Functional Field of View. The Japanese Journal of Psychology, 72, 361-368. https://doi.org/10.4992/jjpsy.72.361

Rosenfeld, J. P. (2011). P300 in Detecting Concealed Information. In B. Verschuere, G. Ben-Shakhar, \& E. Meijer (Eds.), Memory Detection: Theory and Application of the Concealed Information Test (pp. 63-89). Cambridge: Cambridge University Press. https://doi.org/10.1017/CBO9780511975196.005

Rosenfeld, J. P., \& Labkovsky, E. (2010). New P300-Based Protocol to Detect Concealed Information: Resistance to Mental Countermeasures against Only Half the Irrelevant Stimuli and a Possible ERP Indicator of Countermeasures. Psychophysiology, 47, 10021010. https://doi.org/10.1111/j.1469-8986.2010.01024.x

Rosenfeld, J. P., Ozsan, I., \& Ward, A. C. (2017). P300 Amplitude at Pz and N200/N300 Latency at F3 Differ between Participants Simulating Suspect versus Witness Roles in a Mock Crime. Psychophysiology, 54, 640-648. https://doi.org/10.1111/psyp.12823

Rosenfeld, J. P., Shue, E., \& Singer, E. (2007). Single versus Multiple Probe Blocks of P300-Based Concealed Information Tests for Self-Referring versus Incidentally Obtained Information. Biological Psychology, 74, 396-404.

https://doi.org/10.1016/j.biopsycho.2006.10.002

Rozenkrants, B., Olofsson, J. K., \& Polich, J. (2008). Affective Visual Event-Related Potentials: Arousal, Valence, and Repetition Effects for Normal and Distorted Pictures. International Journal of Psychophysiology, 67, 114-123.

https://doi.org/10.1016/j.ijpsycho.2007.10.010

Sarter, M., Givens, B., \& Bruno, J. P. (2001). The Cognitive Neuroscience of Sustained Attention: Where Top-Down Meets Bottom-Up. Brain Research Reviews, 35, 146-160. https://doi.org/10.1016/S0165-0173(01)00044-3

Schupp, H. T., Cuthbert, B. N., Bradley, M. M., Cacioppo, J. T., Ito, T., \& Lang, P. J. (2000). Affective Picture Processing: The Late Positive Potential Is Modulated by Motivational Relevance. Psychophysiology, 37, 257-261.

https://doi.org/10.1111/1469-8986.3720257

Shirasawa, S., Ishida, T., Hakoda, Y., \& Haraguchi, M. (1999). The Effects of Energetic Arousal on Memory Search. The Japanese Journal of Psychonomic Science, 17, 93-99. http://doi.org/10.14947/psychono.KJ00004413571

Silvert, L., Delplanque, S., Bouwalerh, H., Verpoort, C., \& Sequeira, H. (2004). Autonomic Responding to Aversive Words without Conscious Valence Discrimination. International Journal of Psychophysiology, 53, 135-145. https://doi.org/10.1016/j.ijpsycho.2004.03.005

Soskins, M., Rosenfeld, J. P., \& Niendam, T. (2001). Peak-to-Peak Measurement of P300 
Recorded at $0.3 \mathrm{~Hz}$ High Pass Filter Settings in Intraindividual Diagnosis: Complex vs. Simple Paradigms. International Journal of Psychophysiology, 40, 173-180. https://doi.org/10.1016/S0167-8760(00)00154-9

Van den Hout, M. A., De Jong, P., \& Kindt, M. (2000). Masked Fear Words Produce Increased SCRs: An Anomaly for Öhman's Theory of Pre-Attentive Processing in Anxiety. Psychophysiology, 37, 283-288. https://doi.org/10.1111/1469-8986.3730283

Verschuere, B., Crombez, G., Koster, E. H., Van Bockstaele, B., \& De Clercq, A. (2007). Startling Secrets: Startle Eye Blink Modulation by Concealed Crime Information. Biological Psychology, 76, 52-60. https://doi.org/10.1016/j.biopsycho.2007.06.001

Verschuere, B., \& Ben-Shakhar, G (2011). Theory of the Concealed Information Test. In B. Verschuere, G. Ben-Shakhar, \& E. Meijer (Eds.), Memory Detection: Theory and Application of the Concealed Information Test (pp. 128-148). Cambridge: Cambridge University Press. https://doi.org/10.1017/CBO9780511975196.008

Verschuere, B., Ben-Shakhar, G., \& Meijer, E. (2011). Memory Detection: Theory and Application of the Concealed Information Test. Cambridge: Cambridge University Press. https://doi.org/10.1017/CBO9780511975196

Yang, H., Wang, F., Gu, N., Gao, X., \& Zhao, G. (2013). The Cognitive Advantage for One's Own Name Is Not Simply Familiarity: An Eye-Tracking Study. Psychonomic Bulletin \& Review, 20, 1176-1180. https://doi.org/10.3758/s13423-013-0426-z

\section{Submit or recommend next manuscript to SCIRP and we will provide best} service for you:

Accepting pre-submission inquiries through Email, Facebook, LinkedIn, Twitter, etc. A wide selection of journals (inclusive of 9 subjects, more than 200 journals)

Providing 24-hour high-quality service

User-friendly online submission system

Fair and swift peer-review system

Efficient typesetting and proofreading procedure

Display of the result of downloads and visits, as well as the number of cited articles

Maximum dissemination of your research work

Submit your manuscript at: http://papersubmission.scirp.org/

Or contact psych@scirp.org 